\title{
FUNCTIONAL ANALYSIS AND THE METHOD OF HARMONIC BALANCE*
}

\author{
BY \\ C. A. BORGES (Instituto Tecnologico de Aeronautica, Sao Paolo) \\ L. CESARI (University of Michigan, Ann Arbor) \\ AND \\ D. A. SÁNCHEZ (University of California, Los Angeles)
}

1. Introduction. In the Fourier-series expansion of a periodic solution of a nonlinear ordinary differential equation it is often the case that only the components of fundamental frequency have significant amplitude. The principle or method of harmonic balance is that an approximate solution of first-order accuracy is obtained if only the fundamental component is considered, and this solution is adjusted so as to satisfy all terms of fundamental frequency arising in the equation. This is equivalent to replacing the nonlinear equation with a linear equation having the same solution to a first-order approximation (i.e. the method of equivalent linearization). For a discussion of these matters see, for instance, the books by Cunningham [3, Ch. 6] and Kryloff and Bogoliuboff [4, Ch. V].

In this paper we study second-order differential equations describing a pendulum or nonlinear spring with possibly a periodic driving term, and we use the first Galerkin or modified Galerkin approximation to initiate our search for a nearby periodic solution. Then, using techniques of functional analysis developed by Cesari (see [2] for a comprehensive summary of this approach including subsequent work of Hale and others), we show that a certain map associated with the differential equation and the Galerkin approximation has a fixed point. Finally, by studying the associated determining equation, we can show that the fixed point is a periodic solution of the differential equation and lies in a neighborhood of the first Galerkin approximation. More important, the diameter of the neighborhood can be determined and so error bounds on the difference between the first Galerkin approximation and the actual solution can be obtained.

2. The class of equations. We consider the class of second-order scalar ordinary differential equations of the form

$$
\ddot{x}+g(t, x)=0
$$

(or $\ddot{x}+g(x)=0$ in the autonomous case) where $g$ is defined for $|x| \leq A,-\infty<t<\infty$, and for each $x$ is integrable on $[0, T]$ and is $T=2 \pi / \omega$ periodic. We assume that the following symmetry properties are satisfied:

$$
g(-t,-x)=-g(t, x), \quad g\left(\frac{T}{4}+t, x\right)=g\left(\frac{T}{4}-t, x\right) .
$$

* Received May 28, 1973. The research reported herein was supported in part by ITA, São Paulo, Brazil (C.A.B.), US-AFOSR Grant 71-2122 (L.C.), and NSF Grant GP-27972 (D.A.S.). 
Hence a periodic solution, if it exists, will have the Fourier series expansion

$$
x(t) \sim \sum_{j=0}^{\infty} b_{i} \sin (2 j+1) \omega t .
$$

Furthermore, we assume that $g(t, x)$ has the decomposition

$$
g(t, x)=g_{0}(x)+R(t, x)+S(t)
$$

where

(a) $g_{0}(x)=\sigma^{2} x+\sum_{i=1}^{k-1}\left[\alpha_{j} /(2 j+1) !\right] x^{2 i+1}, \sigma \geq 0,|x| \leq A$; in the autonomous case we assume $g_{0}(x)>0$ for $x \in(0, A]$,

(b) $R(t, x)$ is continuous for $|x| \leq A,-\infty<t<\infty$, and

$$
|R(t, x)| \leq \frac{\alpha_{l}}{(2 l+1) !}|x|^{2 l+1}, \quad|x| \leq A, \quad-\infty<t<\infty,
$$

for some integer $l \geq 2 k-1$; in the autonomous case the same condition applies to $R(x)$, and

$$
S(t) \sim \sum_{j=0}^{\infty} A_{2 i+1} \sin (2 j+1) \omega t
$$

Finally we assume that $g(t, x)$ is uniformly Lipschitzian with respect to $x$ with Lipschitz constant $L$ for $|x| \leq A,-\infty<t<\infty$.

3. Some associated operators and a norm. Let $S_{c}$ be the Banach space of all continuous functions $x(t),-\infty<t<\infty, T$-periodic with $T=2 \pi / \omega$ satisfying the symmetry conditions $x(t)=-x(-t), x(T / 4-t)=x(T / 4+t)$, and with norm $\|x\|=$ $\max |x(t)|$. Every element in $S_{c}$ will then have a Fourier-series expansion

$$
x \sim \sum_{k=0}^{\infty} c_{2 k+1} \sin (2 k+1) \omega t, \quad c_{n}=\frac{\omega}{\pi} \int_{0}^{T} x(s) \sin n \omega s d s
$$

and we define the following operators from $S_{c}$ into itself:

$$
P x=c_{1} \sin \omega t, \quad H x=-\sum_{k=0}^{\infty} \frac{c_{2 k+1}}{(2 k+1)^{2} \omega^{2}} \sin (2 k+1) \omega t .
$$

Theorem 1. (a) $P$ is a bounded, linear, idempotent operator and $\|P\| \leq 4 / \pi$; (b) $H$ is a bounded linear operator which commutes with $P$ and $\|H\| \leq \pi / 2 \omega^{2}$; (c) $P H(I-P)=0$.

Proof: Obviously $P$ is linear and idempotent, and from the expression for $c_{1}$ it follows that

$$
\|P x\| \leq \frac{\omega}{\pi}\|x\| \int_{0}^{T}|\sin \omega s| d s=\frac{4}{\pi}\|x\|,
$$

which proves (a). Obviously $H$ is linear and commutes with $P$; also

$$
\|H x\| \leq \omega^{-2}\left(\left|c_{1}\right|+\frac{\left|c_{3}\right|}{3^{2}}+\frac{\left|c_{5}\right|}{5^{2}}+\cdots\right)
$$

and $\left|c_{n}\right| \leq 4 / \pi\|x\|$ for all $n$, so result (b) follows from the fact that $\sum_{k=0}{ }^{\infty}(2 k+1)^{-2}=$ $\pi^{2} / 8$. Part (c) is straightforward.

It is clear that $H(I-P)$ is a linear operator in $S_{c}$ and by the same type of reasoning 
as in (b) above it is not hard to show that $\|H(I-P)\| \leq\left(4 / \pi \omega^{2}\right)\left[\pi^{2} / 8-1\right]=0.29755$ $\omega^{-2}$. But because of the low-order approximation intended, we will need a much better estimate of the bound $\|H(I-P)\|$, which we denote by $k_{c}$.

For $x$ in $S_{c}$,

$$
H x=\int_{0}^{t} d \alpha \int_{T / 4}^{\alpha} x(\beta) d \beta,
$$

and since $c_{1}=(4 \omega / \pi) \int_{0}^{T / 4} x(t) \sin \omega t d t$ and $H(I-P) x=H x+c_{1} \omega^{-2} \sin \omega t$, then

$$
y(t)=H(I-P) x=\int_{0}^{T / 4} K(t, \beta) x(\beta) d \beta
$$

where

$$
\begin{aligned}
K(t, \beta) & =-\beta+\frac{4}{\pi \omega} \sin \omega t \sin \omega \beta, & & 0 \leq \beta \leq t \leq T / 4, \\
& =-t+\frac{4}{\pi \omega} \sin \omega t \sin \omega \beta, & & 0 \leq t \leq \beta \leq T / 4 .
\end{aligned}
$$

Letting $\omega t=\xi, \omega \beta=\eta, X(\xi)=x(\xi / \omega), Y(\xi)=y(\xi / \omega)$, we obtain the equivalent expression

$$
Y(\xi)=\omega^{-2} \int_{0}^{\pi / 2} \bar{K}(\xi, \eta) X(\eta) d \eta
$$

where

$$
\begin{aligned}
\bar{K}(\xi, \eta) & =-\eta+\frac{4}{\pi} \sin \xi \sin \eta, & & 0 \leq \eta \leq \xi \leq \pi / 2, \\
& =-\xi+\frac{4}{\pi} \sin \xi \sin \eta, & & 0 \leq \xi \leq \eta \leq \pi / 2 .
\end{aligned}
$$

By a standard argument (e.g., see [5, pp. 83-84]) we infer that

$$
\|H(I-P)\|=k_{c}=\omega^{-2} k_{0}=\omega^{-2} \max _{0 \leq \xi \leq \pi / 2} \int_{0}^{\pi / 2}|\bar{K}(\xi, \eta)| d \eta
$$

and we must now compute $k_{0}$.

An examination of the kernel $\bar{K}(\xi, \eta)$ in the square $[0, \pi / 2] \times[0, \pi / 2]$ shows that it vanishes only on the $\xi$ and $\eta$ axes and on the curves

$$
\begin{array}{ll}
\eta=\sin ^{-1}\left(\frac{\pi}{4} \frac{\xi}{\sin \xi}\right) & 0 \leq \xi \leq \bar{\xi}, \sin ^{-1}\left(\frac{\pi}{4}\right) \leq \eta \leq \pi / 2, \\
\xi=\sin ^{-1}\left(\frac{\pi}{4} \frac{\eta}{\sin \eta}\right), & \sin ^{-1}\left(\frac{\pi}{4}\right) \leq \xi \leq \pi / 2, \quad 0 \leq \eta \leq \bar{\xi}
\end{array}
$$

where $\bar{\xi}$ is the solution of $\pi / 2=\sin ^{-1}(\pi / 4(\xi / \sin \xi))$. For any value of $\xi$ one can compute the corresponding points on these curves (where $\bar{K}$ changes sign) and then explicitly describe $|\bar{K}(\xi, \eta)|$ and its integral. This was done for a $\xi$-mesh width of $\pi / 2 \times 10^{-2}$ on an IBM 360 and shows that the maximum value is obtained when $\xi=\pi / 2$ and $k_{0}=$ 0.14454 ; hence $k_{c}=0.14454 \omega^{-2}$.

4. The first Galerkin approximation. The equation giving the first Galerkin ap- 
proximation of a $T$-periodic solution to the differential equation (2.1) is

$$
u(a)=\frac{2}{T} \int_{0}^{T}\left[-a \omega^{2} \sin \omega t+g(t, a \sin \omega t)\right] \sin \omega t d t=0
$$

where $T=2 \pi / \omega$. From the decomposition given for $g(t, x)$ we can write this as

$$
u(a)=-a \omega^{2}+A_{1}+\Sigma+\rho=0
$$

where $A_{1}$ is the first Fourier coefficient of $S(t)$ and

$$
\Sigma=\frac{2}{T} \int_{0}^{T} g_{0}(a \sin \omega t) \sin \omega t d t, \quad \rho=\frac{2}{T} \int_{0}^{T} R(t, a \sin \omega t) \sin \omega t d t .
$$

Noting that $|\rho| \leq 2 \alpha_{l}|a|^{2 l+1} /(2 l+1)$ !, we consider instead the modified equation

$$
u_{0}(a)=-a \omega^{2}+A_{1}+\Sigma=0,
$$

and expanding $g_{0}(a \sin \omega t)$ into a trigonometric polynomial and performing the required integration gives

$$
u_{0}(a)=-a \omega^{2}+A_{1}+\sigma^{2} a+2 \sum_{j=1}^{k-1} \frac{\alpha_{i}}{j !(j+1) !}\left(\frac{a}{2}\right)^{2 i+1}=0 .
$$

We call this the modified Galerkin equation and our first Galerkin approximation is $x_{0}=x_{0}(t)=a \sin \omega t$ where $a$ satisfies (4.1). For convenience we assume $0<a \leq A_{0} \leq A$.

In the autonomous case $A_{1}=0$, and then (4.1) defines $\omega=\omega(a)$ satisfying

$$
\omega^{2}=\sigma^{2}+\sum_{j=1}^{k-1} \frac{\alpha_{i}}{j !(j+1) !}\left(\frac{a}{2}\right)^{2 i},
$$

and if $\sigma \neq 0$ then for $|a|$ sufficiently small the right side of (4.1) is positive so $\omega(a)$ is defined and the first Galerkin approximations are $x_{0}=x_{0}(t)=a \sin \omega(a) t$.

If $\sigma=0$ then we observe that

$$
\left|g(x)-g_{0}(x)\right| \leq \frac{\alpha_{l}}{(2 l+1) !}|x|^{2 l+1}
$$

and if $b_{i}=\alpha_{i} /(2 j+1)$ ! and $b_{q}$ is the first nonzero $b_{j}$ then we obtain the estimate

$$
\left|g(x)-b_{a} x^{2 a+3}\right| \leq m\left|b_{a}\right||x|^{2 a+3} D(A)
$$

for $0<x \leq A$, where $D(A)$ is a polynomial in $A$ and $m=\max \left(\left|b_{i}\right| /\left|b_{a}\right|\right), j>q$. For sufficiently small $B, 0<B \leq A, g(x)$ will have the same sign as $b_{a} x^{2 a+1}$ for $0<x<B$, and since we assumed $g(x)>0$ for $0<x<A$, this implies $b_{a}>0$; so we can solve (4.2) for $\omega(a)$ for any $|a|$ sufficiently small, say for $0<a \leq A_{0} \leq A$. This will be implicitly assumed for the autonomous case in the following discussion.

5. The existence of a fixed point. Let $\bar{S}_{c}=\left\{x \mid x \in S_{c},\|x\| \leq A\right\}$ and let the operators $g, F$ and $\mathcal{g}$ from $\bar{S}_{c}$ into $S_{c}$ be defined by

$$
\begin{aligned}
& g x=g(t, x(t)), \quad F x=-H(I-P) g x, \\
& g x=P x+F x=P x-H(I-P) g x .
\end{aligned}
$$

Given any number $b$ such that $0<b \leq A_{0}$, let $x_{b}=x_{b}(t)=b \sin \omega t$ (or $b \sin \omega(b) t$ in the autonomous case) and define $\Delta(t, b)=H(I-P) g x_{b}$. Given our first Galerkin 
approximation $x_{0}=x_{0}(t)=a \sin \omega t$ (the dependence of $\omega$ on $a$ in the autonomous case is implicitly understood), let $c, d>0$ be two constants such that $0<c<d$ and $|b-a| \leq c$. We can assume that $0<a+c \leq A_{0}$ by adjusting $A_{0}$ if necessary. Then define

$$
S_{c}^{b}=\left\{x \mid x \in S_{c}, P x=x_{b},\left\|x-x_{0}\right\| \leq d\right\}
$$

and $S_{c}{ }^{b}$ is nonempty since it contains $x_{b}$, it is a complete metric space, and if $a+d \leq A$ then $S_{c}{ }^{b} \subset \bar{S}_{c}$ since $x \in S_{c}{ }^{b}$ implies $\|x\| \leq\left\|x-x_{0}\right\|+\left\|x_{0}\right\| \leq a+d$.

Theorem 2. Suppose $k_{c} L<1$ and there exist constants $c, d$ satisfying $0<c<d$, $a+d \leq A$ and $|b-a| \leq c$ where $x_{b}=b \sin \omega t$ is given. Furthermore, suppose

$$
c+\gamma_{c} \leq\left(1-k_{c} L\right) d
$$

where $\gamma_{\mathrm{o}}=\max \|\Delta(t, \alpha)\|,|\alpha| \leq A_{0}$. Then $\mathrm{g}$ maps $S_{c}{ }^{b}$ into itself and is a contraction; hence it has a unique fixed point $y$. This fixed point satisfies

$$
\left\|y-x_{b}\right\| \leq k_{c} L d+\gamma_{c} \leq d-c .
$$

Proof: For $x \in S_{c}{ }^{b}$ let $y=\mathfrak{d} x=P x-H(I-P) g x$ and since $P y=P x=x_{b}$ and $\Delta(t, a)=H(I-P) g x_{0}$ we have

$$
\begin{aligned}
\left\|y-x_{0}\right\| & =\left\|x_{b}-x_{0}-H(I-P)\left(g x-g x_{0}\right)-\Delta(t, a)\right\| \\
& \leq\left\|x_{b}-x_{0}\right\|+\|H(I-P)\|\left\|g x-g x_{0}\right\|+\|\Delta(t, a)\| \\
& \leq|b-a|+k_{c} L\left\|x-x_{0}\right\|+\gamma_{c} \leq c+k_{c} L d+\gamma_{c} \leq d,
\end{aligned}
$$

and so $\mathcal{J}$ maps $S_{c}{ }^{b}$ into itself, and it is certainly continuous. If $y_{1}=\mathscr{J} x_{1}, y_{2}=\mathscr{J} x_{2}$ then

$$
\left\|y_{1}-y_{2}\right\|=\left\|H(I-P)\left(g x_{1}-g x_{2}\right)\right\| \leq k_{c} L\left\|x_{1}-x_{2}\right\|
$$

and $k_{c} L<1$ implies $\mathcal{J}$ is also a contraction. Hence by Banach's fixed-point theorem it has a unique fixed point $y$. Finally we note that $y$ satisfies

$$
\begin{aligned}
\left\|y-x_{b}\right\| & =\left\|-H(I-P)\left(g y-g x_{0}\right)-\Delta(t, a)\right\| \\
& \leq k_{c} L d+\gamma_{c} \leq d-c,
\end{aligned}
$$

and so our conclusion follows.

We observe that since the fixed point $y \in S_{c}^{b}$ of $g$ satisfies $y=P y-H(I-P) g y$ then it also satisfies $\ddot{y}=P \ddot{y}-(I-P) g y$ or equivalently

$$
\ddot{y}(t)+g(t, y(t))=P(\ddot{y}(t)+g(t, y(t)))=U(b) \sin \omega t .
$$

Since $y(t) \sim b \sin \omega t+\sum_{i=1}^{\infty} b_{2 i+1}(b) \sin (2 j+1) \omega t$ and $g(t, y(t)) \sim \sum_{i=0}{ }^{\infty} \beta_{2 j+1}(b)$ $\cdot \sin (2 j+1) \omega t$, we see that if

$$
U(b)=-\omega^{2} b+\beta_{1}(b)=0
$$

then $y$ is a $T$-periodic solution of the differential equation (2.1).

Eq. (5.3) is called the determining equation and we must show there exists a number $b$ such that $|b-a| \leq c$ and which satisfies (5.3) to complete our proof of existence.

6. The solution of the determining equation. Letting $x_{b}=x_{b}(t)=b \sin \omega t$ as before, suppose $g\left(t, x_{b}(t)\right)=\sum_{i=0}{ }^{\infty} \tilde{\beta}_{2 j+1}(b) \sin (2 j+1) \omega t$ and then 


$$
u(b)=\frac{2}{T} \int_{0}^{T}\left[\ddot{x}_{b}(t)+g\left(t, x_{b}(t)\right)\right] \sin \omega t d t=-\omega^{2} b+\widetilde{\beta}_{1}(b) .
$$

It follows that

$$
\begin{aligned}
|U(b)-u(b)| & =\left|\beta_{1}(b)-\tilde{\beta}_{1}(b)\right|=|| P\left[g(t, y(t))-g\left(t, x_{b}(t)\right)\right] \| \\
& \leq \frac{4}{\pi} L\left\|y-x_{b}\right\| \leq \frac{4}{\pi} L\left(k_{c} L d+\gamma_{c}\right) .
\end{aligned}
$$

Furthermore, we have

$$
\begin{aligned}
u(b)= & {\left[-b \omega^{2}+A_{1}+\sigma^{2} b+2 \sum_{j=1}^{k-1} \frac{\alpha_{j}}{j !(j+1) !}\left(\frac{b}{2}\right)^{2 j+1}\right] } \\
& +\left[\frac{2}{T} \int_{0}^{T} R(t, b \sin \omega t) \sin \omega t d t\right]=u_{0}(b)+\eta(b)
\end{aligned}
$$

where $u_{0}(a)=0$, and

$$
\begin{aligned}
|\eta(b)| & \leq \frac{2}{T} \frac{\left.\alpha_{l}|| b\right|^{2 l+1}}{(2 l+1) !} \int_{0}^{T}|\sin \omega t|^{2 l+1} d t \\
& \leq \frac{4}{\pi} \frac{\alpha_{l} A_{0}{ }^{2 l+1}}{[1 \cdot 3 \cdots(2 l+1)]^{2}}=\eta .
\end{aligned}
$$

Since we can solve for the expression $\sigma^{2}-\omega^{2}$ from the modified Galerkin approximation (4.2), we can obtain the expression

$$
u_{0}(b)=A_{1}-b\left\{\frac{A_{1}}{a}+\sum_{i=1}^{k-1} \frac{\alpha_{j}}{j !} \cdot\left[\left(\frac{a}{2}\right)^{2 i}-\left(\frac{b}{2}\right)^{2 i}\right]\right\}
$$

and from this obtain expressions for $u_{01}=u_{0}(a-c)$ and $u_{02}=u_{0}(a+c)$. We assume these have opposite signs, which will certainly be the case if $a$ is a simple root of $u_{0}(a)$ since

$$
u_{0}(a \pm c)= \pm c u_{0}{ }^{\prime}(a)+o(|c|)
$$

and let $Q=\min \left(\left|u_{01}\right|,\left|u_{02}\right|\right)$.

Theorem 3. Given the assumptions above, suppose that

$$
\eta<Q
$$

and

$$
\frac{4}{\pi} L\left(k_{c} L d+\gamma_{c}\right)<Q-\eta .
$$

Then there exists a number $b$ such that $|b-a| \leq c$ and $b$ satisfies the determining equation $U(b)=0$.

Proof: We need only show that $U(b)$ has opposite signs at the points $b=a \pm c$. Assume that $u_{01}<0<u_{02}$; hence $u_{0}(a-c) \leq-Q<0<Q \leq u_{0}(a+c)$ and $u(a \pm c)$ $=u_{0}(a \pm c)+\eta(a \pm c)$. Then for $b=a+c$

$$
\begin{aligned}
U(b)=u_{0}(b)+(U(b)-u(b))+\eta(b) & \geq u_{0}(b)-|U(b)-u(b)|-\eta \\
& \geq Q-\frac{4}{\pi} L\left(k_{c} L d+\gamma c\right)-\eta>0
\end{aligned}
$$


and in a similar manner one can show $U(b)<0$ for $b=a-c$, which gives the desired result since $U(b)$ is continuous.

Coroltary: Under the assumptions given and the hypotheses of Theorems 2 and 3 , the fixed point

$$
y \sim b \sin \omega t+b_{3} \sin 3 \omega t+\cdots
$$

of the man $\mathcal{I}$ in $S_{c}{ }^{b}$ is a $T$-periodic solution of (2.1) where $b$ satisfies $|b-a| \leq c$ and

$$
\left\|y-x_{0}\right\| \leq d, \quad\left\|y-x_{b}\right\| \leq k_{c} L d+\gamma_{c} .
$$

Here $x_{11}=x_{0}(t)=a \sin \omega t$ is the first Galerkin approximation and $x_{b}=x_{b}(t)=b \sin \omega t$.

7. An example. We consider the differential equation

$$
\ddot{x}+\sin x=-\frac{1}{6} \sin t
$$

and let

$$
g(t, x)=x-\frac{1}{6} x^{3}+\frac{1}{120} x^{5}+R(x)+\frac{1}{6} \sin t .
$$

Then $L=1, \sigma^{2}=\omega^{2}=1, A_{1}=\frac{1}{6}, g_{0}(x)=x-\frac{1}{6} x^{3}+\frac{1}{12} x^{5}, k_{c}=0.14454$ and for $|x| \leq A,|R(x)| \leq A^{7} / 5040$. The modified Galerkin equation is

$$
u_{0}(a)=\frac{1}{192}\left(32-24 a^{3}+a^{5}\right)=0
$$

which has a root $a=1.12053$. Since we assume $0<c<a$ this implies $Q=\left|u_{0}(a-c)\right|$, and substituting gives

$$
Q=0.42979 c-0.34692 c^{2}+0.05960 c^{3}-0.02918 c^{4}+0.00521 c^{5} .
$$

Furthermore, we have

$$
\begin{aligned}
\Delta(t, b)= & H(I-P) g(t, b \sin t) \\
= & \left(-\frac{1}{216} b^{3}+\frac{1}{3456} b^{5}\right) \sin 3 t-\frac{1}{48000} b^{5} \sin 5 t \\
& +H(I-P) \sum_{i=3}^{\infty} \frac{(-1)^{i}(b \sin t)^{2 j+1}}{(2 j+1) !},
\end{aligned}
$$

and, for $|b| \leq A_{0}$,

$$
\begin{aligned}
\|\Delta(t, b)\| \leq \frac{1}{216} A_{0}{ }^{3} & +\left(\frac{1}{3456}+\frac{1}{4800}\right) A_{0}{ }^{5} \\
& +k_{c}\left[\sinh A_{0}-A_{0}-\frac{1}{6} A_{0}{ }^{3}-\frac{1}{120} A_{0}{ }^{5}\right]=\gamma_{c},
\end{aligned}
$$

and

$$
|\eta(b)| \leq \frac{4}{\pi \cdot 11025} A_{0}{ }^{7}=\eta
$$

Then for $A_{0}=1.3$,

$$
\gamma_{c}=0.01151, \quad \eta=0.00072
$$


and since $1-k_{c} L=0.85546$, the inequalities to be satisfied by $c$ and $d$ are

$$
\begin{aligned}
& c+0.01151 \leq 0.85546 d, \quad 0.00072<Q, \\
& \frac{4}{\pi}(0.14454 d+0.01151)<Q-0.00072 .
\end{aligned}
$$

The inequalities are equivalent to finding a value of $c$ for which

$$
0<P(c)=1.16645 c-1.88508 c^{2}+0.32385 c^{3}-0.15857 c^{4}+0.02830 c^{5}-0.09509 \text {. }
$$

The smallest such positive $c$ is $c=0.09626$ and the corresponding value of $d$ is $d=$ 0.12597. It follows then that (7.1) has a $2 \pi$-periodic solution

$$
y=y(t) \sim b \sin t+b_{3} \sin 3 t+\cdots
$$

where

$$
|b-1.12053| \leq 0.09626 \text { and } \| y-1.12053 \sin t \mid \leq 0.12957 \text {. }
$$

8. Final remarks and acknowledgments. The initial efforts of this research were made by coauthor C. A. Borges in his unpublished doctoral dissertation [1]; there he also considers the existence of periodic solutions in the Hilbert space of square-integrable, $T$-periodic functions satisfying the given symmetry conditions. The operators $P$ and $H$ are the same and one easily obtains the norm

$$
\|H(I-P)\|=\frac{1}{9} \omega^{-2}
$$

by an analysis of the eigenvalues of $H(I-P)$. The statements of the corresponding theorems showing that $T$ is a contraction and that the determining equation has a solution are almost identical to the ones given here and will not be stated.

For some classes of differential equations of the type discussed the estimate given in Sec. 6 for

$$
|U(b)-u(b)|=\left|\beta_{1}(b)-\widetilde{\beta}_{1}(b)\right|
$$

is not sharp enough to insure that the required inequalities (5.1) and (6.2) are satisfied. In particular, this may occur when $L=1$, and so sharper estimates of the above difference are required.

The authors wish to gratefully acknowledge the assistance of Professors J. Krupp and G. Richter in the programming necessary to compute the norm $k_{c}$.

\section{REFERENCES}

[1] C. A. Borges, Periodic solutions of nonlinear differential equations: existence and crror bounds, Ph.D. dissertation, University of Michigan, 1963

[2] L. Cesari, Functional analysis and differential equations, Studies in Applied Mathematics 5, SIAM, 1969, 143-155

[3] W. J. Cunningham, Introduction to nonlinear analysis, McGraw-Hill, New York, 1958

[4] N. Kryloff and N. Bogoliuboff, Introduction to nonlinear mechanics (translated by S. Lefschetz), Annals of Mathematics Study No. 11, Princeton University Press, Princeton, 1947

[5] L. Liusternik and V. Sobolev, Elements of functional analysis (translated by A. Labarre Jr., H. Izbicki and H. Crowley), Frederick Ungar, New York, 1961 